\title{
TANGENCY PORTFOLIOS IN THE LP SOLVABLE PORTFOLIO SELECTION MODELS
}

\author{
Reza Keykhaei $^{1}$ And Mohamad Taghi JahandideH ${ }^{1}$
}

\begin{abstract}
A risk measure in a portfolio selection problem is linear programming (LP) solvable, if it has a linear formulation when the asset returns are represented by discrete random variables, i.e., they are defined by their realizations under specified scenarios. The efficient frontier corresponding to an LP solvable model is a piecewise linear curve. In this paper we describe a method which realizes and produces a tangency portfolio as a by-product during the procedure of tracing out of the efficient frontier of risky assets in an LP solvable model, when our portfolio contains some risky assets and a riskless asset, using nonsmooth optimization methods. We show that the test of finding the tangency portfolio can be limited only for two portfolios. Also, we describe that how this method can be employed to trace out the efficient frontier corresponding to a portfolio selection problem in the presence of a riskless asset.
\end{abstract}

Keywords. Linear programming, LP solvable portfolio selection models, subgradient, tangency portfolio, Aneja-Nair method.

Mathematics Subject Classification. 90C05, 90C29.

\section{INTRODUCTION}

In the original mean-risk Markowitz's portfolio selection problem [8], the risk is measured by the variance, which leads the problem to a quadratic programing optimization problem. After that, some alternative risk measures have been

Received February 6, 2012. Accepted May 22, 2012.

1 College of Mathematical Sciences, Isfahan University of Technology, 84156-83111 Isfahan, Iran. r.keykhaei@math.iut.ac.ir; jahandid@cc.iut.ac.ir 
considered to represent the portfolio selection problem as a linear programming optimization problem. A risk measure is linear programming (LP) solvable, if it has a linear formulation when the asset returns are represented by discrete random variables, i.e., they are defined by their realizations under specified scenarios. Konno and Yamazaki [5] presented their LP solvable optimization model using mean absolute deviation as the measure of risk, which is an $l_{1}$ risk function. Young [14] introduced a minimax LP solvable portfolio selection model based on risk defined by the worst case scenario. Teo and Yang [11] and Cai et al. [3] presented their minimax portfolio selection models by bi-criteria linear programming problems using $l_{\infty}$ risk functions. Also, we can refer to LP solvable models considered by Yitzhaki [13] and Rockafellar and Uryasev [9]. Mansini et al. [7] investigated a systematic properties and computational comparison for LP solvable models.

Tütüncü [12] introduced a simple modification of Markowitz's critical line method which is commonly used for generating the mean-variance efficient frontier to determine the tangency portfolio which maximizes the ratio $\left(\rho-r_{f}\right) / \sigma$ between all feasible portfolios, where $\rho$ and $\sigma$ denote the mean and the standard deviation of the return of a feasible portfolio and $r_{f}$ is the return of the riskless asset. Indeed he has shown how this modification can be used to produce the tangency portfolio as a by-product during the procedure of tracing out of the efficient frontier of risky assets.

In the spirit of Tütüncü's work, we suggest an approach which determines the location of a tangency portfolio for mean-risk LP solvable portfolio selection problems. Also, the tangency portfolio can be obtained as a by-product in the procedure of generating the efficient frontier of risky assets. Indeed, by this method, it is enough to realize a tangency portfolio between two efficient portfolios, but not all of them, which can be useful in large-scale portfolio selection problems. Moreover, we show that how this method can be employed to trace out the efficient frontier corresponding to a portfolio selection problem in the presence of a riskless asset without involving the free asset in the problem directly.

The organization of the paper is as follows. In Section 2, a portfolio selection problem is formulated as a LP solvable problem. In Section 3, we study some property of a tangency portfolio employing nonsmooth optimization tools. In Section 4, we describe how we can produce a tangency portfolio as a by-product during the procedure of tracing out the efficient frontier of risky assets using a standard bicriteria linear programming optimization method. A numerical example is given in Section 5.

\section{LP SOLVABLE MODELS}

Consider a portfolio consists of $n \geq 2$ risky assets with the random returns $r_{1}, \ldots, r_{n}$ and the mean vector $\overline{\mathbf{r}}=\left(\bar{r}_{1}, \ldots, \bar{r}_{n}\right)^{\prime}$. We denote each portfolio by the vector of asset weights $\mathbf{x}=\left(x_{1}, \ldots, x_{n}\right)^{\prime} \in \mathbb{R}^{n}$, where each $x_{i}$ is the weight allocated to the $i$ th asset. Here $\mu(\mathbf{x})=\overline{\mathbf{r}}^{\prime} \mathbf{x}$ and $\varphi(\mathbf{x})$ denote the mean of return and the risk associated to the portfolio, respectively. We consider the following LP 
solvable portfolio selection problem corresponding to a desired expected return $\rho$ :

$$
\min _{\mathbf{x}}\left\{\varphi(\mathbf{x}): \overline{\mathbf{r}}^{\prime} \mathbf{x}=\rho, \mathbf{A}_{0} \mathbf{x}=\mathbf{b}_{0}, \mathbf{C}_{0} \mathbf{x} \geq \mathbf{d}_{0}\right\}
$$

A portfolio $\mathbf{x}$ is said to be feasible if it satisfies the last two conditions in problem (2.1).

We assume that problem (2.1) can be stated or approximated by an equivalent linear programming as follows:

problem (ELP):

$$
\begin{array}{ll}
\min _{\mathbf{X}} & \bar{\varphi}(\mathbf{X})=\mathbf{k}^{\prime} \mathbf{X} \\
\text { s.t. } & \overline{\mathbf{R}}^{\prime} \mathbf{X}=\rho, \\
& \mathbf{A X}=\mathbf{b} \\
& \mathbf{C X} \geq \mathbf{d},
\end{array}
$$

where $\mathbf{k}, \overline{\mathbf{R}} \in \mathbb{R}^{N}, \mathbf{b} \in \mathbb{R}^{m}, \mathbf{d} \in \mathbb{R}^{p}, \mathbf{A}$ is an $m \times N$, and $\mathbf{C}$ is a $p \times N$ matrix over $\mathbb{R}$. In the following each decision vector $\mathbf{X} \in \mathbb{R}^{N}$ describes our adapted portfolio and in the general case, a portfolio $\mathbf{X}$ is said to be feasible if it satisfies the last two conditions in problem (ELP). By the Kuhn-Tucker optimality conditions, $\mathbf{X}^{*}$ is a (primal) solution of problem (ELP), if and only if, there exist vectors $\lambda_{\rho} \in \mathbb{R}$, $\lambda_{\mathbf{b}} \in \mathbb{R}^{m}$ and $\lambda_{\mathbf{d}} \in \mathbb{R}^{p}$ such that:

$$
\begin{gathered}
\mathbf{k}-\lambda_{\rho} \overline{\mathbf{R}}-\mathbf{A}^{\prime} \lambda_{\mathbf{b}}-\mathbf{C}^{\prime} \lambda_{\mathbf{d}}=0 \\
\overline{\mathbf{R}}^{\prime} \mathbf{X}^{*}=\rho, \quad \underset{\mathbf{A X}}{\mathbf{A}}=\mathbf{b}, \quad \lambda_{\mathbf{d}}^{\prime}\left(\mathbf{C X}^{*}-\mathbf{d}\right)=0, \\
\mathbf{C X}^{*} \geq \mathbf{d}, \quad \lambda_{\mathbf{d}} \geq 0
\end{gathered}
$$

For any expected return $\rho$, let $\Omega(\rho)$ denotes the set of all primal-dual solutions $\left(\mathbf{X}^{*}, \Lambda\right)$ of problem (ELP), satisfying $(2.2)$, where $\Lambda=\left(\lambda_{\rho}, \lambda_{\mathbf{d}}, \lambda_{\mathbf{d}}\right)$. We call $\lambda_{\rho}$ an optimal reward multiplier, which it is not necessarily unique.

A feasible portfolio $\mathbf{X}$ is said to be efficient if there exists no feasible portfolio $\mathbf{Y}$ such that

$$
\mathbf{k}^{\prime} \mathbf{Y} \leq \mathbf{k}^{\prime} \mathbf{X}, \quad \overline{\mathbf{R}}^{\prime} \mathbf{Y} \geq \overline{\mathbf{R}}^{\prime} \mathbf{X}
$$

and at least one of the inequalities holds strictly. Let $\rho_{\min }$ be the expected return of an efficient portfolio which has the minimum obtainable risk. Also let $\rho_{\max }$ be the highest obtainable expected return of feasible portfolios. Actually, any efficient portfolio has expected return $\rho \in\left[\rho_{\min }, \rho_{\max }\right]$. The value of $\rho_{\max }$ can be infinite. The graph which plots the risk of any efficient portfolio against its expected return is called the efficient frontier. As [12], we define function

$$
\begin{gathered}
\phi:\left[\rho_{\min }, \rho_{\max }\right] \longrightarrow \mathbb{R} \\
\rho \mapsto \mathbf{k}^{\prime} \mathbf{X}^{*} ;
\end{gathered}
$$

where $\left(\mathbf{X}^{*}, \Lambda\right) \in \Omega(\rho)$. Indeed the efficient frontier is the set

$$
E=\left\{(\rho, \phi(\rho)) ; \rho \in\left[\rho_{\min }, \rho_{\max }\right]\right\} .
$$


$\phi$ is a convex function and the efficient frontier is a piecewise linear convex curve. Note that $\phi$ is not necessarily smooth. Indeed, for any $\rho$, it might be more than one subgradient of $\phi$ at $\rho$. Remind that the scaler $c$ is a subgradient of $\phi$ at $\rho$, if and only if

$$
\phi\left(\rho_{1}\right) \geq \phi(\rho)+c\left(\rho_{1}-\rho\right)
$$

for any $\rho_{1} \in\left[\rho_{\min }, \rho_{\max }\right]$. Also, the set of all such scalers, denoted by $\partial \phi(\rho)$, is called the subdifferential of $\phi$ at $\rho$. We can see that there is a close connection between subdifferential of $\phi$ at $\rho$ and the set of optimal reward multipliers of problem (ELP):

Proposition 2.1. $\partial \phi(\rho)$ is equal with the set of optimal reward multipliers of problem (ELP).

Proof. See Chapter VII, Theorem 3.3.2 of [4].

\section{TANGENCY PORTFOLiO}

In this section we consider a new asset which is riskless with return $r_{f}$ to be added in our portfolio described in the last section. We assume that $r_{f}<\rho_{\min }$. We consider such risks which in the presence of a riskless asset, the risk of each convex combination of the riskless asset and a feasible risky portfolio can be represented by the similar convex combination of corresponding risks, i.e., each portfolio can be represented by a point on the line called the capital allocation line (CAL) connecting the feasible risky portfolio and the riskless asset in the mean-risk plane. When borrowing is allowed, the efficient frontier is the straight line (which dominates the other obtained lines) known as capital market line (CML), passing from the $r_{f}$ on mean axis and a portfolio on the efficient frontier of risky assets known as the tangency portfolio. The nature of CML implies that its slope is a subgradient of $\phi$ at the mean return of the tangency portfolio in the mean-risk framework. In fact the CML has the lowest slope between all CALs; and tangency portfolio maximizes the ratio

$$
S(\mathbf{x})=\frac{\mathbf{x}^{\prime} \overline{\mathbf{r}}-r_{f}}{\varphi(\mathbf{x})}
$$

among all feasible portfolios. When standard deviation is considered as the measure of risk, $S(\mathbf{x})$ indicates the Sharpe ratio [10].

Note that, since $\phi$ is not necessarily strictly convex, it is possible that there exist more than one tangency portfolio. In the following let $\rho(T)=\left[\underline{\rho}_{T}, \bar{\rho}_{T}\right]$ (possibly the single point $\rho_{T}$ ) denotes the set of the expected returns of all tangency portfolios.

Lemma 3.1. Let $\left(\mathbf{X}^{*}, \Lambda\right) \in \Omega(\rho)$, for which

$$
\lambda_{\rho}=\frac{\mathbf{k}^{\prime} \mathbf{X}^{*}}{\rho-r_{f}},
$$

then $\mathbf{X}^{*}$ is a tangency portfolio. 
Proof. The proof follows from Proposition 2.1 and the simple fact that the slope of the capital market line is $\phi(\rho) /\left(\rho-r_{f}\right)$.

For $\left(\mathbf{X}^{*}, \Lambda\right) \in \Omega(\rho)$ we define

$$
\theta(\Lambda):=r_{f} \lambda_{\rho}+\mathbf{b}^{\prime} \lambda_{\mathbf{b}}+\mathbf{d}^{\prime} \lambda_{\mathbf{d}}
$$

The sign of the above expression has a key role in our approach to determine the location of a tangency portfolio.

Theorem 3.2. Let $\left(\mathbf{X}^{*}, \Lambda\right) \in \Omega(\rho)$. If $\theta(\Lambda)=0$, then $\rho \in \rho(T)$. Also, if $\rho \in$ $\left(\bar{\rho}_{T}, \rho_{\max }\right]\left(\rho \in\left[\rho_{\min }, \underline{\rho}_{T}\right)\right)$, then $\theta(\Lambda)<0(\theta(\Lambda)>0)$.

Proof. Let $\left(\mathbf{X}^{*}, \Lambda\right) \in \Omega(\rho)$. Multiply the first row of $(2.2)$ by $\mathbf{X}^{* \prime}$ and use the second row of $(2.2)$ to obtain

$$
\bar{\varphi}\left(\mathbf{X}^{*}\right)=\lambda_{\rho} \rho+\mathbf{b}^{\prime} \lambda_{\mathbf{b}}+\mathbf{d}^{\prime} \lambda_{\mathbf{d}}=\lambda_{\rho}\left(\rho-r_{f}\right)+\theta(\Lambda) .
$$

If $\theta(\Lambda)=0$, then

$$
\lambda_{\rho}=\frac{\phi(\rho)}{\rho-r_{f}} .
$$

Then $\rho \in \rho(T)$ by Lemma 3.1. If $\rho \in\left(\bar{\rho}_{T}, \rho_{\max }\right]$, we have

$$
\lambda_{\rho}>\frac{\phi(\rho)}{\rho-r_{f}} .
$$

Thus, by $(3.2)$ we get $\theta(\Lambda)<0$. The proof of the rest of the claim is similar.

Corollary 3.3. Let $\left(\mathbf{X}^{*}, \Lambda\right) \in \Omega\left(\rho_{\max }\right)\left(\Omega\left(\rho_{\min }\right)\right)$. If $\theta(\Lambda)>0(\theta(\Lambda)<0)$, then $\rho_{\max } \in \rho(T)\left(\rho_{\min } \in \rho(T)\right)$.

Proof. Let $\left(\mathbf{X}^{*}, \Lambda\right) \in \Omega\left(\rho_{\max }\right)$ and $\theta(\Lambda)>0$. Suppose that, on the contrary, $\rho_{\max } \notin$ $\rho(T)$. Then, by Theorem 3.2 we have $\theta(\Lambda)<0$, which contradicts the assumption. Thus, $\rho_{\max } \in \rho(T)$. The other statement can be proved similarly.

\section{Applying Aneja-Nair method}

We employ the method of Aneja and Nair [1], initially proposed for a bi-objective (transportation) optimization problem, in order to tracing out the efficient frontier of problem (ELP). Their procedure generates all efficient extreme points on the objective space rather than on the decision space (the method was adapted in [6] to find an approximation of the efficient extreme points). For a given pair of efficient extreme point, a single objective problem (see problem $(i)$ ) is solved and this problem leads to either a new efficient extreme point or changes the direction of search in the objective space.

We summarize the Aneja-Nair method (corresponding to our case) as follows: We label the mean axis and the risk axis by $Z_{1}$ and $Z_{2}$, respectively, in the 


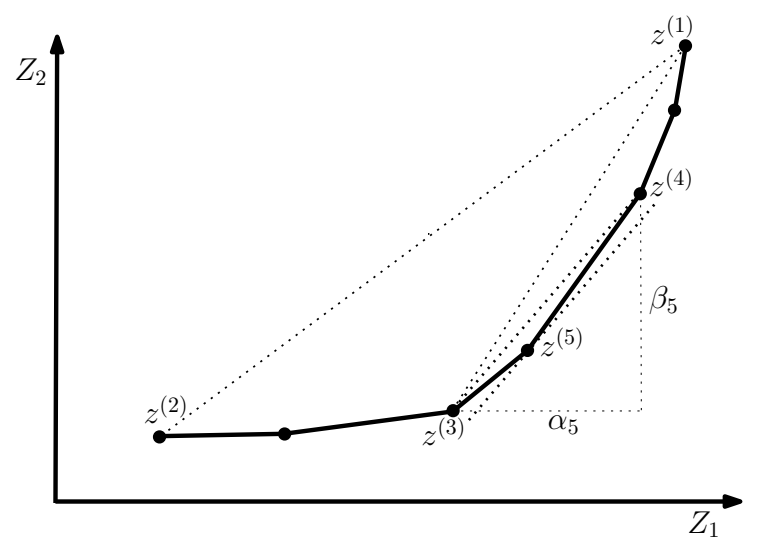

Figure 1. The efficient frontier of problem (ELP) computed by Aneja-Nair method.

objective space (see Fig. 1). The procedure first finds two efficient extreme points $z^{(1)}$ corresponding to a portfolio which has the lowest risk among the portfolios with the highest obtainable return; and $z^{(2)}$ corresponding to a portfolio which has the highest return among the portfolios with the lowest obtainable risk. In fact the procedure first solves problem (ELP) for $\rho=\rho_{\max }$ and $\rho=\rho_{\min }$. To obtain the $i$ 'th efficient extreme point $(i \geq 3)$, using the two efficient extreme points $z^{(r)}=\left(z_{1}^{(r)}, z_{2}^{(r)}\right)$ and $z^{(s)}=\left(z_{1}^{(s)}, z_{2}^{(s)}\right)$ considered by the algorithm, the following problem is used:

problem $(i)$ :

$$
\begin{array}{ll}
\min _{\mathbf{X}} & \alpha_{i} Z_{2}(\mathbf{X})-\beta_{i} Z_{1}(\mathbf{X})=\alpha_{i} \mathbf{k}^{\prime} \mathbf{X}-\beta_{i} \overline{\mathbf{R}}^{\prime} \mathbf{X} \\
\text { s.t. } & \mathbf{A X}=\mathbf{b} \\
& \mathbf{C X} \geq \mathbf{d},
\end{array}
$$

where $\alpha_{i}=\left|z_{1}^{(r)}-z_{1}^{(s)}\right|$ and $\beta_{i}=\left|z_{2}^{(r)}-z_{2}^{(s)}\right|$. If there are alternative optima, a portfolio with highest return is chosen. Now, problem $(i)$ produces a new efficient extreme point, if the location of a chosen solution dose not coincide with $z^{(r)}$ or $z^{(s)}$ in the objective space. The algorithm terminates when no extreme point or no improving direction is available.

Let $\Omega_{i}$ denotes the set of all primal-dual solutions $\left(\mathbf{X}^{*}, \hat{\Lambda}\right)$ of Problem $(i)$, where $\hat{\Lambda}=\left(\hat{\lambda}_{\mathbf{b}}, \hat{\lambda}_{\mathbf{d}}\right)$ in which $\hat{\lambda}_{\mathbf{b}}$ and $\hat{\lambda}_{\mathbf{d}}$ are the Lagrangian multipliers corresponding to the first and the second constraint, respectively. Note that applying the SIMPLEX method enables us to extract the dual solutions from its tableau, see [2]. Moreover, most of LP solvers in mathematical softwares such as MATLAB, compute dual solutions as well as primal solutions. 
Lemma 4.1. Let $\left(\mathbf{X}^{*}, \hat{\Lambda}\right) \in \Omega_{i}$. Then $\left(\mathbf{X}^{*}, \lambda_{\rho}, \lambda_{\mathbf{b}}, \lambda_{\mathbf{d}}\right) \in \Omega(\rho)$, where $\rho=Z_{1}\left(\mathbf{X}^{*}\right)$, $\lambda_{\rho}=\beta_{i} / \alpha_{i}$ and $\left(\lambda_{\mathbf{b}}, \lambda_{\mathbf{d}}\right)=\left(1 / \alpha_{i}\right) \hat{\Lambda}$.

Proof. Let $\left(1 / \alpha_{i}\right) \hat{\Lambda}=\left(\lambda_{\mathbf{b}}, \lambda_{\mathbf{d}}\right)$. Since $\left(\mathbf{X}^{*}, \hat{\Lambda}\right) \in \Omega_{i}$ then

$$
\begin{gathered}
\alpha_{i} \mathbf{k}-\beta_{i} \overline{\mathbf{R}}-\mathbf{A}^{\prime} \hat{\lambda}_{\mathbf{b}}-\mathbf{C}^{\prime} \hat{\lambda}_{\mathbf{d}}=0 \\
\mathbf{A X}^{*}=\mathbf{b}, \quad \hat{\lambda}_{\mathbf{d}}^{\prime}\left(\mathbf{C X}^{*}-\mathbf{d}\right)=0 \\
\mathbf{C X}^{*} \geq \mathbf{d}, \quad \hat{\lambda}_{\mathbf{d}} \geq 0 .
\end{gathered}
$$

Now, by positivity of $\alpha_{i}$ and (4.1) we have

$$
\begin{gathered}
\mathbf{k}-\left(\beta_{i} / \alpha_{i}\right) \overline{\mathbf{R}}-\mathbf{A}^{\prime} \lambda_{\mathbf{b}}-\mathbf{C}^{\prime} \lambda_{\mathbf{d}}=0 \\
\mathbf{A X}^{*}=\mathbf{b}, \quad \lambda_{\mathbf{d}}^{\prime}\left(\mathbf{C X}^{*}-\mathbf{d}\right)=0 \\
\mathbf{C X}^{*} \geq \mathbf{d}, \quad \lambda_{\mathbf{d}} \geq 0
\end{gathered}
$$

Considering (4.2) and the fact that $\overline{\mathbf{R}}^{\prime} \mathbf{X}^{*}=Z_{1}\left(\mathbf{X}^{*}\right)$, we see that $\left(\mathbf{X}^{*}, \beta_{i} / \alpha_{i}, \lambda_{\mathbf{b}}, \lambda_{\mathbf{d}}\right)$ satisfies the Kuhn-Tucker optimality conditions for problem (ELP) for the expected return $\rho=\overline{\mathbf{R}}^{\prime} \mathbf{X}^{*}$. This completes the proof.

Note. Obviously $\beta_{i} / \alpha_{i}$ is equal to the slope of the line connected two points $z^{(r)}$ or $z^{(s)}$ and the slope of the optimal line proposed by Problem $(i)$. Note that Lemma 4.1 and Proposition 2.1 confirm the last case (see Fig. 1).

For $\left(\mathbf{X}^{*}, \hat{\Lambda}\right) \in \Omega_{i}$ let

$$
\hat{\theta}(\hat{\Lambda})=\beta_{i} r_{f}+\mathbf{b}^{\prime} \hat{\lambda}_{\mathbf{b}}+\mathbf{d}^{\prime} \hat{\lambda}_{\mathbf{d}}
$$

Now we have the following version of Theorem 3.2.

Theorem 4.2. Let $\left(\mathbf{X}^{*}, \hat{\Lambda}\right) \in \Omega_{i}$. If $\hat{\theta}(\hat{\Lambda})=0$, then $Z_{1}\left(\mathbf{X}^{*}\right) \in \rho(T)$. Also, if $Z_{1}\left(\mathbf{X}^{*}\right) \in\left(\bar{\rho}_{T}, \rho_{\max }\right]\left(Z_{1}\left(\mathbf{X}^{*}\right) \in\left[\rho_{\min }, \underline{\rho}_{T}\right)\right)$, then $\hat{\theta}(\hat{\Lambda})<0(\hat{\theta}(\hat{\Lambda})>0)$.

Proof. The proof is a straightforward result of Lemma 4.1 and Theorem 3.2.

Note that since the efficient frontier is piecewise linear, we can relate a tangency portfolio to an efficient extreme point. Using the Aneja-Nair method and Theorem 4.2 and Corollary 3.3, we can recognize a tangency portfolio and produce it as a by-product in the procedure of tracing out the efficient frontier corresponding to risky assets. At the first step, we examine the sign of $\theta(\Lambda)$ at $z^{(1)}$ and $z^{(2)}$ corresponding to $\rho=\rho_{\max }$ and $\rho=\rho_{\min }$, respectively. If $\theta(\Lambda) \geq 0$, then $z^{(1)}$ can be considered as a tangent point. If $\theta(\Lambda) \leq 0$ at $z^{(2)}$, then $\rho_{\min } \in \rho(T)$. Otherwise, If we met an efficient extreme point in which $\hat{\theta}(\hat{\Lambda})=0$, then this point corresponds to a tangency portfolio. In the last case, if we recognize two adjacent efficient extreme points for which $\hat{\theta}(\hat{\Lambda})$ takes different sign, then the point which maximizes the ratio (3.1) corresponds to a tangency portfolio. In fact, this method enables us to compare the value of (3.1) only between two points. This property is quite useful, specially for large-scale portfolio selection problems which produce a large 
number of efficient extreme points. Obviously, the procedure can be stopped after finding a tangency portfolio if the identifying of a tangency portfolio is interested only.

We can employ this method to trace out the efficient frontier of a portfolio selection problem for which a riskless asset is involved. In the following we consider a portfolio as a tangency portfolio if its return is equal to $\bar{\rho}_{T}$. In fact we can lead the procedure of finding the location of a tangency portfolio to the direction for which $\hat{\theta}(\hat{\Lambda})$ takes negative sign. By this, we can give up the generator efficient extreme points $z^{(r)}$ and $z^{(s)}$ (and other efficient extreme points located between them) for which $\hat{\theta}(\hat{\Lambda})$ is positive in both points and focus on pairs $\left(z^{(r)}, z^{(s)}\right)$ whit nonpositive sign of $\hat{\theta}(\hat{\Lambda})$. Obviously the efficient extreme points located on the left side of a tangent point should be removed at the end of the procedure.

\section{Illustrative EXAMPle}

In this section the method is described by an example considering the MAD model proposed by Konno and Yamazaki [5], where risk is measured by absolute deviation. Using historical data, the MAD model measures the risk as follows:

$$
\varphi(\mathbf{x})=E\left(\left|\sum_{i=1}^{n} r_{i} x_{i}-E\left(\sum_{i=1}^{n} r_{i} x_{i}\right)\right|\right)=\frac{1}{T} \sum_{t=1}^{T}\left|\sum_{i=1}^{n}\left(r_{i t}-\bar{r}_{i}\right) x_{i}\right|,
$$

where $r_{i t}$ is the observed rate of return of asset $i$ at time period $t(t=1, \ldots, T)$ and $\bar{r}_{i}=\left(\sum_{t=1}^{T} r_{i t}\right) / T$. Let $a_{i t}=r_{i t}-\bar{r}_{i}$ for $t=1, \ldots, T$ and $i=1, \ldots, n$. Now the MAD portfolio selection problem

$$
\min _{\mathbf{x}}\left\{\varphi(\mathbf{x})=\frac{1}{T} \sum_{t=1}^{T}\left|\sum_{i=1}^{n} a_{i t} x_{i}\right|: \overline{\mathbf{r}}^{\prime} \mathbf{x}=\rho, \mathbf{A}_{0} \mathbf{x}=\mathbf{b}_{0}, \mathbf{C}_{0} \mathbf{x} \geq \mathbf{d}_{0}\right\}
$$

is equivalent to the following LP problem (see Konno and Yamazaki [5]):

$$
\begin{aligned}
& \min _{\mathbf{X}=(\mathbf{x}, \mathbf{y})} \bar{\varphi}(\mathbf{X})=\frac{1}{T} \sum_{t=1}^{T} y_{t} \\
& \text { s.t. } \quad \overline{\mathbf{r}}^{\prime} \mathbf{x}=\rho, \\
& \mathbf{A}_{0} \mathbf{x}=\mathbf{b}_{0} \text {, } \\
& \mathbf{C}_{0} \mathbf{x} \geq \mathbf{d}_{0} \text {, } \\
& y_{t}+\sum_{i=1}^{n} a_{i t} x_{i} \geq 0, \quad t=1, \ldots, T, \\
& y_{t}-\sum_{i=1}^{n} a_{i t} x_{i} \geq 0, \quad t=1, \ldots, T \text {. }
\end{aligned}
$$

Example 5.1. In this example we consider the MAD model with feasible region $S=\left\{\mathbf{x} \in \mathbb{R}^{n}: \sum_{i=1}^{n} x_{i}=1 ; x_{i} \geq 0, i=1, \ldots, n\right\}$ and the following data set 
TABLE 1. Efficient extreme points corresponding to Example (5.1).

\begin{tabular}{c|cccccccccc}
\hline & $z^{(1)}$ & $z^{(5)}$ & $z^{(6)}$ & $z^{(4)}$ & $z^{(7)}$ & $z^{(3)}$ & $z^{(10)}$ & $z^{(9)}$ & $z^{(11)}$ & $z^{(8)}$ \\
\hline Mean (\%) & 4.72 & 4.51 & 4.1921 & 3.882 & 3.6713 & 3.6452 & 3.4383 & 3.2321 & 3.1479 & 2.9074 \\
Risk (\%) & 2.898 & 2.4252 & 1.7943 & 1.2306 & 0.9856 & 0.9564 & 0.782 & 0.628 & 0.5818 & 0.4886 \\
$\theta(\Lambda) ; \hat{\theta}(\hat{\Lambda})\left(10^{-5}\right)$ & -4351.55 & -29.87 & -20.89 & -33.02 & -3.62 & -27.61 & -3.14 & -3.47 & -0.41 & -0.18 \\
\hline & $z^{(14)}$ & $z^{(13)}$ & $z^{(12)}$ & $z^{(16)}$ & $z^{(15)}$ & $z^{(17)}$ & $z^{(2)}$ & & \\
\hline Mean(\%) & 2.7109 & 2.611 & 2.4089 & 2.1452 & 1.8963 & 1.8247 & 1.7422 & \\
$\operatorname{Risk}(\%)$ & 0.4256 & 0.4003 & 0.3631 & 0.3175 & 0.2863 & 0.2835 & 0.2831 & \\
$\theta(\Lambda) ; \hat{\theta}(\hat{\Lambda})\left(10^{-5}\right)$ & 0.19 & 0.6 & 2.36 & 1.13 & 1.59 & 0.43 & 284.45 & \\
\hline
\end{tabular}

of the asset rate of returns (expressed in \%) for $n=11$ assets over $T=10$ time periods:

$$
\left(\begin{array}{cccccccccc}
2.04 & 0.84 & 0.19 & 2.38 & 4.02 & 1.94 & 0.89 & -0.19 & 0.04 & -0.02 \\
-0.25 & 0.03 & 1.26 & 2.82 & 1.77 & 0.87 & 0.74 & 2.99 & 2.9 & -0.13 \\
5.4 & -4.8 & 0.12 & 3.54 & 5.91 & 3.39 & 0.08 & 6.62 & 2.78 & -6.83 \\
5.85 & 5.53 & 3.67 & 4.57 & 2.1 & 2.15 & 1.89 & -2.18 & -2.45 & -3.63 \\
-3.84 & 0.64 & 4.28 & 4.49 & 3.74 & 3.56 & 2.92 & 4.09 & 7.34 & 6.87 \\
0.61 & -0.67 & 2.77 & 5.42 & 6.67 & 11.93 & 5.17 & 1.68 & 6.32 & 7.3 \\
0.68 & 8.31 & 5.7 & -1.75 & -1.77 & 6.24 & -0.67 & 0.29 & -6.26 & -3.43 \\
5.87 & 5.07 & 7 & 6.8 & 5.09 & -3.03 & -4.37 & -5.22 & -4.39 & -1.99 \\
1.02 & 2.59 & 0.49 & -0.51 & 0.97 & 4.47 & 6.94 & -0.06 & 0.56 & 1.37 \\
7.44 & 5.6 & 4.42 & 3.69 & 4.78 & 4.74 & 0.06 & -1.27 & 1.67 & -0.19 \\
3.6 & 7.51 & 0.53 & -2.71 & -0.95 & 3.22 & 5.13 & 3.24 & 5.56 & 2.33
\end{array}\right),
$$

where the $i$ 'th row corresponds to the observations of the $i$ 'th asset. The primaldual solutions are computed using SIMPLEX method in MATLAB. The efficient extreme points obtained by Aneja-Nair method and their corresponding amount of $\hat{\theta}(\hat{\Lambda})$ corresponding to $r_{f}=.015$ are represented in Table 1 (Also, see Fig. 2). Note that $\theta(\Lambda)$ is only computed for $z^{(1)}$ and $z^{(2)}$ and $\hat{\theta}(\hat{\Lambda})$ is computed for the other efficient extreme points. The $i$ 'th efficient point is shown by $z^{(i)}$ and is obtained from two closest points with the lower labels at its left and its right sides. For example $z^{(8)}$ is obtained from $z^{(3)}$ and $z^{(2)}$. As we can see, the sign of $\hat{\theta}(\hat{\Lambda})$ is changed between $z^{(8)}$ and $z^{(14)}$. Then, at the end of the procedure of finding efficient extreme points, we can also introduce $z^{(8)}$ as the tangency portfolio. Note that, if we are only interested in the tangency portfolio, then we can stop the procedure after $z^{(14)}$ and introduce the points $z^{(1)}-z^{(8)}$ and $z^{(0)}=(.015,0)$ as the set of efficient extreme points corresponding to the MAD portfolio selection problem consisting of the above $n$ risky assets (with the feasible region $S$ ) and a riskless asset whit $r_{f}=.015$ for which borrowing is not allowed. 


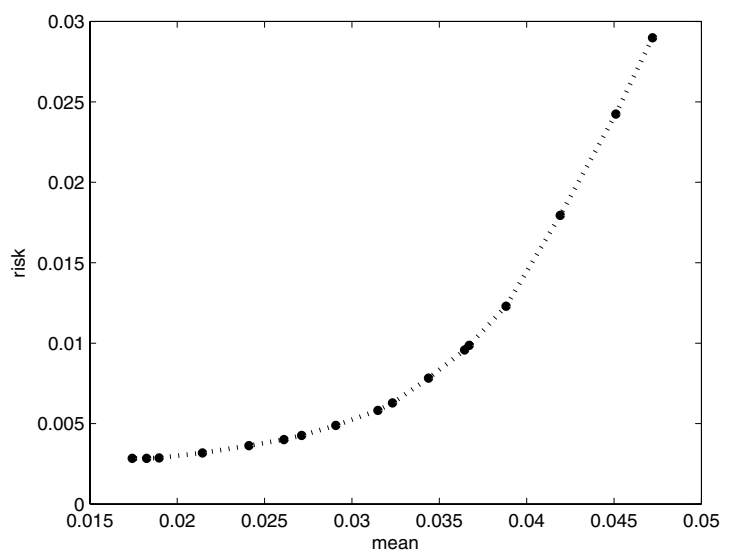

FiguRE 2. The efficient frontier of Example (5.1).

\section{REFERENCES}

[1] Y.P. Aneja and K.P.K. Nair, Bicriteria transportation problem. Manage. Sci. 25 (1979) $73-78$.

[2] M.S. Bazaraa, J.J. Jarvis and H.D. Sherali, Linear programming and network flows, 3rd edition. John Wiley \& Sons, New York (2005).

[3] X.Q. Cai, K.L. Teo, X.Q. Yang, and X.Y. Zhou, Portfolio optimization under a minimax rule. Manage. Sci. 46 (2000) 957-972.

[4] J.-B. Hiriart-Urruty and C. Lemaréchal. Convex analysis and minimization algorithms I. Springer, Berlin, Heidelberg, New York (1993).

[5] H. Konno and H. Yamazaki, Mean-absolute deviation portfolio optimization model and its applications to Tokyo stock market. Manage. Sci. 37 (1991) 519-529.

[6] T. Lust and J. Teghem, Two-phase pareto local search for the biobjective traveling salesman problem. J. Heuristics 16 (2010) 475-510.

[7] R. Mansini, W. Ogryczak and M.G. Speranza, On LP solvable models for portfolio selection. Informatica 14 (2003) 37-62.

[8] H.M. Markowitz, Portfolio selection. J. Financ. 7 (1952) 77-91.

[9] R.T. Rockafellar and S. Uryasev, Optimization of conditional value-at-risk. J. Risk 2 (2000) 21-41.

[10] W.F. Sharpe, The Sharpe ratio. J. Portfolio Manage. Fall (1994) 49-58.

[11] K.L. Teo and X.O. Yang, Portfolio selection problem with minimax type risk function. Ann. Oper. Res. 101 (2001) 333-349.

[12] R.H. Tütüncü, A note on calculating the optimal risky portfolio. Finance Stochastics $\mathbf{5}$ (2001) 413-417.

[13] S. Yitzhaki, Stochastic dominance, mean variance, and Ginis mean difference. Amer. Econ. Rev. 72 (1982) 178-185.

[14] M.R. Young, A minimax portfolio selection rule with linear programming solution. Manage. Sci. 44 (1998) 673-683. 